\section{Schnupfen an Bord?}

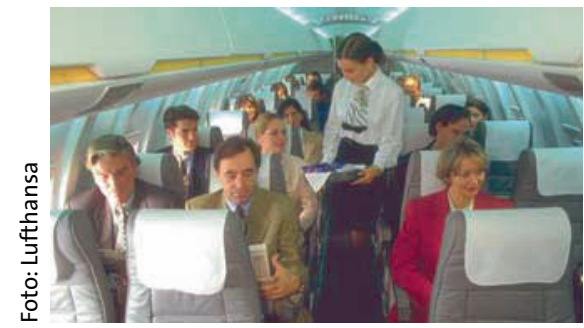

Di ie Ventilationssysteme neuer Verkehrsflugzeuge stehen in Verdacht, die Verbreitung von Infektionskrankheiten zu fördern. Um Treibstoff zu sparen führen moderne Lüftungssysteme den Passagieren nämlich nicht mehr 100\% Frischluft zu, sondern wälzen etwa 50\% der Kabinenluft erneut um. Experimente amerikanischer Wissenschaftler haben diesen Verdacht jedoch nicht bestätigen können. Die Forscher befragten 1.100 Passagiere unterschiedlich ausgestatteter Flugzeuge direkt vor und sieben Tage nach einem zweistündigen Flug, ob bei ihnen Symptome wie eine laufende Nase, Kopf- oder Halsweh aufgetreten waren. Die Auswertung ergab keinen Unterschied zwischen Passagieren, die in Flugzeugen mit Frischluft- bzw. Rezirkulationssystemen reisten.

mwe

Zitter JN et al. Jama 2002; $288: 483-6$

\title{
Berufskrankheit Popcorn-Lunge
}

$\mathrm{n}$ einer amerikanischen Fabrik zur Herstellung von Mikrowellen-Popcorn fiel auf, dass acht ehemalige Arbeiter an einer Bronchiolitis obliterans litten. Daraufhin nahmen US-Mediziner vom National Institute for Occupational Safety and Health die Belegschaft der Fabrik genauer unter die Lupe. Verglichen mit nationalen Referenzdaten waren bei den 117 Arbeitern chronischer Husten sowie Kurzatmigkeit um

\section{Asthma durch Impfung?}

mpfkritiker verdächtigen die üblichen Schutzimpfungen bei Säuglingen und Kleinkindern immer wieder, unvermutete Nebenwirkungen auszulösen. Zumindest was das Risiko anbelangt, durch eine Vakzination an Asthma zu erkranken, können Frank Destefano und seine Kollegen von den Centers of Disease Control and Prevention in Atlanta jetzt Entwarnung geben. In einer Kohortenstudie dokumentierten sie die Daten von rund 170.000 Kindern, die sie von Geburt an über mehrere Jahre hinweg begleite- den Faktor 2,6 erhöht, Asthma und chronische Bronchitis 2-mal so häufig und Atemwegsobstruktionen traten 3,3-mal öfter auf. Die Wissenschaftler verdächtigen als Auslöser das flüchtige Diacetyl, ein Keton, das aufgrund seines Buttergeschmacks der Popcorn-Gewürzmischung beigefügt wird.

Kreiss K et al. N Engl J Med 2002; 347: 330-8 ten. Die US-Wissenschaftler konnten keinen signifikanten Zusammenhang zwischen einer Asthmaerkrankung und einer der routinemäßig bei Kleinkindern eingesetzten Vakzinationen herstellen. Bei einem relativen Risiko zwischen 0,92 für die Diphterie-, Tetanusund Pertussis-Impfung und 1,20 für die Hepatitis-Impfung konnten sie alle der neun gängigen Immunisierungen entlasten.

Destefano F et al. Pediatr Infect Dis J

2002; 21: 498-504

\section{Endspurt für den Lungentag}

\begin{abstract}
A m 28. September es soweit: Im Rahmen des 5. Deutschen Lungentages will die „Aktion gesunde Lunge“, an der auch die DGAI beteiligt ist, die Bevölkerung für die Frühsymptome von Atemwegs- und Lungenkrankheiten sensibilisieren und Informationen über Erkennungs- und Behandlungsmöglichkeiten aufzeigen. Dazu finden bundesweit Informationsveranstaltungen in Fußgängerzonen und Arztpraxen statt. Einen genauen Fahrplan gibt's im Internet.
\end{abstract} Lungenen ag

\section{Wiedervereinigung auch in der Luft}

W issenschaftler vom GSF-Forschungszentrum Neuherberg haben im Rahmen einer Langzeituntersuchung zwischen 1992 und 1999 die Eltern von insgesamt 5.000 Schulkindern in den Kreisen Bitterfeld, Hettstedt und Zerbst nach möglichen Atemwegserkrankungen ihrer Sprösslinge gefragt. Die Auswertung der Fragebögen zeigte einen deutlichen Zusammenhang zwischen der Häufigkeit der Erkrankungen und den mittleren Konzentrationen an Schwebstaub und Schwefeldioxid in der Luft. Diese Luftschadstoffe hatten sich während des Untersuchungszeitraum durch die Schließung zahlreicher Industrieanlagen nach der Wiedervereinigung um bis zu $90 \%$ verringert. Insgesamt sank die Anzahl nicht allergischer respiratorischer Erkrankungen seit 1992 um mehr als ein Drittel und er-

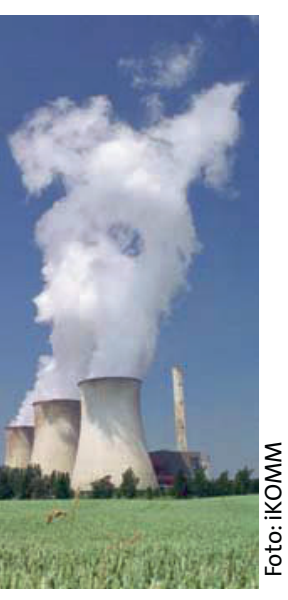
reicht damit westdeutsches Niveau. $n z$

Heinrich J et al. Epidemiology 2002; 13: 394-401 